J. Math. Soc. Japan

Vol. 25, No. 2, 1973

\title{
On universal embeddings in matrix rings
}

\author{
By S. A. AMITSUR
}

(Received March 17, 1972)

Given a ring $R$ and an integer $n \geqq 1$ we have shown in [1] that there exists a commutative ring $S$ and a homomorphism $\rho: R \rightarrow M_{n}(S)$, the matrix rings over $S$, such that every other homomorphism $\sigma: R \rightarrow M_{n}(K)$ with $K$ commutative, is induced by a homomorphism $\eta: S \rightarrow K$. The category of rings considered in [1] was the category of rings not necessarily containing a unit, but $S$ was taken to be a ring with a unit. It seems that that was not the natural assumption on $S$ and in fact this caused an incomplete proof in the last section of [1]. In the present note, a different ring $S_{0}$ is obtained with the aid of the preceding ring $S$ which is the natural universal object in the category of rings (not necessarily containing a unit). This is applied to give a characterization of ring whose irreducible representations are of $\operatorname{dim} \geqq n$. Finally, the results obtained yield a universal splitting ring of central separable algebras. A byresult is a criterion for a set of matrices to generate the full matrix ring.

\section{$\S 1$. Notations and remarks.}

These will follow the notations of [1]: $k$ will denote a fixed commutative ring with a unit. All rings considered here will be $k$-algebras and all homomorphism will be $k$-homomorphism.

$M_{n}(R)$ will denote the $n \times n$ matrix ring over a ring $R$, and $M_{n}(\eta): M_{n}(R)$ $\rightarrow M_{n}(S)$ will denote the homomorphism of the matrix rings induced by a homomorphism $\eta: R \rightarrow S$.

$k[x]$ will denote the free ring generated over $k$ by a set $\left\{x_{i}\right\}$ of noncommutative indeterminates. Denote by $S_{i}=\left(\xi_{\alpha \beta}^{i}\right) \alpha, \beta=1,2, \cdots, n$ generic matrices of order $n$ over $k$, where the $\xi_{\alpha \beta}^{i}$ are commutative indeterminates over $k . \Delta=k[\xi]$ will denote the ring of commutative polynomials in the $\xi$ 's; and $k[X]$ will be the subring of $M_{n}(\Delta)$ generated by the generic matrices.

The notation $\Delta^{0}=k^{0}[\xi], k^{0}[x], k^{0}[X]$ will stand for the corresponding rings of polynomial with zero as the free coefficient. 


\section{$\S 2$. Generators of matrix rings.}

It was shown in [1], p. 27 that there exists a maximal ideal $T$ in the polynomial ring $\Delta$ such that $k^{0} \Delta[X] \supseteqq \mathscr{M}_{n}(T)$. This ideal $T$ can be used as a tool to test if a set of matrices $\left\{A_{\lambda}\right\}$ in a matrix ring $\mathscr{M}_{n}(H)$ over a field $H \supseteqq k$ generate the whole matrix ring $\mathscr{M}_{n}(H)$. This is carried out as follows:

To the set of matrices $A_{\lambda}=\left(a_{i k}^{\lambda}\right)$ we make correspond a homomorphism $\varphi: \Delta \rightarrow H$ defined by $\varphi\left(\xi_{i k}^{\lambda}\right)=a_{i k}^{\lambda}$, and extend it to a homomorphism $\mathscr{M}_{n} \varphi$ : $\mathscr{M}_{n}(\Delta) \rightarrow \mathscr{M}_{n}(H)$, by which the generic matrices $X_{\lambda}$ correspond to the matrix $A_{i}$. Then

THEOREM 1. The set $\left\{A_{\lambda}\right\}$ generates $\mathscr{M}_{n}(H)$ if and only if $\varphi(T) \neq 0$.

Proof. If $\varphi(T) \neq 0$, then the algebra $H^{0}\left[A_{\lambda}\right]$ generated by the $A_{\lambda}$ 's will satisfy :

$$
H^{0}\left[A_{\lambda}\right] \supseteqq \mathscr{M}_{n} \varphi\left[k^{0}[X] \Delta\right] \supseteqq \mathscr{M}_{n} \varphi\left(\mathscr{M}_{n}(T)\right)=\mathscr{M}_{n}(\varphi(T)) .
$$

Hence, for each pair $(i, k)$ there exists $0 \neq t \in \varphi(T)$ such that $t c_{i k} \in H^{0}\left[A_{\lambda}\right]$ where $c_{i k}$ are the standard matrix units of $\mathscr{M}_{n}(H)$. Since $t \neq 0$ in the field $H$, it follows that also $c_{i k}=t^{-1}\left(t c_{i k}\right) \in H^{0}\left[A_{\lambda}\right]$ and consequently, $H^{0}\left[A_{\lambda}\right] \supseteqq \mathscr{M}_{n}(H)$ which clearly implies that $H^{0}\left[A_{\lambda}\right]=\mathscr{M}_{n}(H)$ since $H^{0}\left[A_{\lambda}\right] \cong M_{n}(H)$.

Conversely, let $H^{0}\left[A_{\lambda}\right]=\mathscr{M}_{n}(H)$, which is equivalent to the fact that $H k^{0}\left[A_{\lambda}\right]=\mathscr{M}_{n}(H)$. Hence, there exist $n^{2}$ polynomials $\left\{f_{i}[X]\right\}$ in $k^{0}[X]$ such that the set $\left\{f_{i}\left[A_{\lambda}\right]\right\}$ is a base of $\mathscr{M}_{n}(H)$. We follow now the proof of Theorem 5 of [1]: If $\operatorname{tr}(\cdot)$ denotes the reduced trace, then since $\left\{f_{i}\left[A_{\lambda}\right]\right\}$ is a base it follows that $0 \neq \delta=\operatorname{det}\left(\operatorname{tr}\left[f_{i}\left(A_{\lambda}\right) f_{j}\left(A_{\lambda}\right)\right]\right)$. Now note that the reduced trace commutes with $\varphi$; hence,

$$
\delta=\operatorname{det}\left(\operatorname{tr}\left[\varphi\left(f_{i}\left[A_{\lambda}\right] f_{j}\left[A_{\lambda}\right]\right)\right]\right)=\varphi \operatorname{det}\left(\operatorname{tr} f_{i}[X] f_{j}[X]\right)
$$

and if $d=\operatorname{det}\left(\operatorname{tr} f_{i}[X] f_{j}[X]\right)=0$ then $\varphi(d)=\delta \neq 0$. It remains now to show that $d \in T$.

Indeed, $d$ being the determinant of traces and non zero implies that the set $\left\{f_{i}[X]\right\}$ is a base in $\mathscr{M}_{n}(\Omega)$ (ฏ $\mathscr{M}_{n}(\Delta)$ ) where $\Omega$ is the ring of quotients of $\Delta$. Thus in particular we have: $c_{i k}=\sum f_{\lambda}[X] u_{\lambda, i k}$ with $u_{\lambda, i k} \in \Omega$. Multiplying this equality by $f_{\mu}[x]$ and taking the trace we get $\Sigma \operatorname{tr}\left(f_{\mu}[X] f_{\lambda}[x]\right) u_{\lambda, i k}$ $=h_{\mu, i k}=\sum \operatorname{tr}\left(f_{\mu}[X] c_{i k}\right) \in \Delta$. Eliminating these equations by Cramer's rule, it follows that $d u_{\lambda, i k}=g_{\lambda, i k} \in \Delta$. Hence, $d c_{i k}=\sum f_{\lambda}[X] d u_{\lambda, i k} \in k^{0}[X] \Delta$, which means that $d \in T$. q.e.d.

\section{$\S 3$. The universal embedding.}

In [1] we have defined a universal embedding $\rho: R \rightarrow \mathscr{M}_{n}(S)$ for an arbitrary ring $R$, and where $S=S(n ; R)$ is a commutative ring with a unit. In 
this section we replace $S$ by a subring $S_{0}$ which also has universal properties and which is more natural in the category of rings which do not necessarily contain a unit.

THEOREM 2. There exists a commutative ring $S_{0}$ and a homomorphism $\rho_{0}$ : $R \rightarrow \mathscr{M}_{n}\left(S_{0}\right)$ such that:

(i) (a) $S_{0}$ is generated by the entries $\left\{\rho_{0}(r)_{i k} ; r \in R\right\}$.

(b) For any $\sigma: R \rightarrow M_{n}(K), K$ a commutative ring (not necessarily with a unit)-there exists a homomorphism $\eta: S_{0} \rightarrow K$ such that the induced map $\mathscr{M}_{n}(\eta): \mathscr{M}_{n}\left(S_{0}\right) \rightarrow \mathscr{M}_{n}(K)$ satisfies the relation $\mathscr{M}_{n}(\eta) \rho_{0}=\sigma$.

(ii) $S_{0}$ is uniquely determined (up to an isomorphism) by the existence of a map $\rho_{0}: R \rightarrow \mathscr{M}_{n}\left(S_{0}\right)$ satisfying (a) and (b). Furthermore, $\rho_{0}$ is determined up to a multiple of an isomorphism of $S_{0}$; and given $S_{0}, \rho_{0}$ and $\sigma$ then $\eta$ and $\mathscr{M}_{n}(\eta)$ are uniquely determined.

(iii) If $\rho: R \rightarrow \mathscr{M}_{n}(S)$ is the universal embedding of $R$ (as given in [1] Theorem 1$)$, then $S_{0}$ can be chosen to be an ideal in $S$, such that $S / S_{0} \cong k$ and $\rho_{0}$ is the map $\rho$ restricted to $S_{0}$. Furthermore $\rho(R) S \cong \mathscr{M}_{n}\left(S_{0}\right) \cong \mathscr{M}_{n}(S)$.

PROOF. The proof of (ii) is the same as that of (ii) of Theorem 1 of [1] with the observation that no unit is required here.

The proof of (i) can be obtained by following similar lines to the proof of the same theorem, but we rather prove (iii) from which (i) will follow.

To this end we recall the definition of $S$ of [1]: Let $\left\{r_{i}\right\}$ be a set of generators of $R$, then $k^{0}[x] / \mathfrak{p} \cong R$ by the mapping $x_{i} \rightarrow r_{i}$. We also considered the homomorphism: $k^{0}[x] / \mathfrak{p} \rightarrow k^{0}[X] / P$ induced by the map $x_{i} \rightarrow X_{i}$, and where $P$ is the image of $\mathfrak{p}$. Finally $P$ generates an ideal $\{P\}=\mathscr{M}_{n}(I)$ in $\mathscr{M}_{n}(\Delta)$ with $I$ an ideal in $\Delta=k[\xi]$ (in which a unit exists). The universal ring $S$ was shown to be $\Delta / I$ and $\rho$ was the composite map:

$$
R \rightarrow k^{0}[x] / \mathfrak{p} \rightarrow k^{0}[X] / P \rightarrow \mathscr{M}_{n}(\Delta) /\{P\} \rightarrow \mathscr{M}_{n}(\Delta / I)=\mathscr{M}_{n}(S) .
$$

We now set $S_{0}=\Delta^{0} / I$, i. e. the ideal mod $I$ generated by all polynomials in the $\xi$ 's with zero free coefficient. Clearly $S_{0}$ is an ideal in $S$. Note also that $I \subseteq \Delta^{0}$, since the correspondence: $r_{i} \rightarrow 0$ induces the homomorphism: $R \rightarrow 0$, which implies the ideal $\mathfrak{p}$ defined by the representation $R=k^{0}[x] / \mathfrak{p}$ is in $k^{0}[x]$. Therefore, the image $P$ will contain in its matrices entries with zero as constant coefficients, i.e. $P \subseteq \mathscr{M}_{n}\left(\Delta^{0}\right)$ and hence $I \subseteq \Delta^{0}$. This, in particular, implies that $S / S_{0} \cong(\Delta / I) /\left(\Delta^{0} / I\right)=\Delta / \Delta^{0}=k$. Furthermore, since $\mathscr{M}_{n}\left(S_{0}\right) \supseteqq$ the image of $k^{0}[X] / \mathfrak{p}$, it follows that $\rho(R) \cong \mathscr{M}_{n}\left(S_{0}\right)$ and in fact even $\rho(R) S \subseteq \mathscr{M}_{n}\left(S_{0}\right)$. Thus $\rho$ induces a homomorphism $\rho_{0}: R \rightarrow \mathscr{M}_{n}\left(S_{0}\right)$ which will satisfy (i). Indeed: For any $r_{i} \in R, \rho_{0}\left(r_{\lambda}\right)_{i k}=\xi_{i k}^{\lambda}+I$, and $S_{0}=\Delta^{0} / I$ is generated by $\xi_{i k}^{\lambda}+I$ i. e. by $\left\{\rho_{0}(r)_{i k} ; r \in R\right\}$. If $\sigma: R \rightarrow \mathscr{M}_{n}(K)$; then if $K$ does not contain a unit, extend $K$ to $K^{*}$ by adding a unit and consider the composite 
$\sigma^{*}: R \rightarrow \mathscr{M}_{n}(K) \rightarrow \mathscr{M}_{n}\left(K^{*}\right)$. It follows now by Theorem 1] of [1] that there exists a homomorphism $\eta: S \rightarrow K^{*}$ such that $\left(\mathscr{M}_{n} \eta\right) \rho=\sigma^{*}$. Note that $\sigma^{*}(R)$ $=\sigma(R)$ and $\rho(R)=\rho_{0}(R)$ in $\mathscr{M}_{n}\left(S_{0}\right)$; hence, if $\eta_{0}$ is $\eta$ reduced to $S_{0}$ we get $\left(\mathscr{M}_{n} \eta_{0}\right) \rho_{0}(r)=\sigma(r)$ for every $r \in R$ and consequently $\left(\mathscr{M}_{n} \eta_{0}\right) \rho_{0}=\sigma$ as required in (b). This completes the proof.

\section{$\S 4$. Representations.}

We are now able to obtain the correct set-up for the statement of Theorem 5 of [1]:

Recall that an irreducible representation of $R$ of dimension $n$ is a homomorphism $\varphi: R \rightarrow \mathscr{M}_{n}(F), F_{a}$ commutative field and such that $\varphi(R) F=\mathscr{M}_{n}(F)$.

THEOREM 3. Let $R$ be either a ring with a unit, or a finitely generated $k$-algebra such that $R^{2}=R$ then: all irreducible representations of $R$ are of dimension $\geqq n$ if and only if $\rho(R) S=\mathscr{M}_{n}\left(S_{0}\right)$ i.e. the subring generated by $S_{0}$ and $\rho(R)$ is $\mathscr{M}_{n}\left(S_{0}\right)$.

If this holds, $R$ has no representation of lower degree, and then $S_{0} \neq 0$ if and only if $R$ has a non-zero representation (not necessarily irreducible) of dimension $n$, and then furthermore, all its representations of $\operatorname{dim} n$ are irreducible.

Proof. Consider the ideal $T \cong \Delta$ of Theorem 1 and note that its definition implies that $T \cong \Delta^{0}$. Let $T_{0}=T+I / I \cong \Delta^{0} / I=S_{0}$.

Assume that every irreducible representation of $R$ is of $\operatorname{dim} \geqq n$. Let $\mathfrak{q}$ be any prime ideal in $S_{0}$ containing $T_{0}$, then we have a representation $\sigma$ of $R$ :

$$
R \rightarrow \mathscr{M}_{n}\left(S_{0}\right) \rightarrow \mathscr{M}_{n}\left(S_{0} / \mathfrak{q}\right) \rightarrow \mathscr{M}_{n}(H)
$$

where $H$ is the field of quotients of the domain $S_{0} / \mathfrak{q}$. This representation cannot be irreducible since the corresponding $\varphi$ of Theorem 1 is in our case given by: $\varphi: r \rightarrow \rho_{0}(r)+\mathscr{M}_{n}(\mathfrak{q})$ in $\mathscr{M}_{n}(H)$, but then since $T_{0} \subseteq q$ it follows that $\varphi(T)=0$. Thus, the images of the generators $\left\{r_{\lambda}\right\}$ do not contain a base of $\mathscr{M}_{n}(H)$.

If it were $\sigma(R) \neq 0$, then since $R^{2}=R$ it follows that $\sigma(R)$ is not nilpotent. Since $\sigma(R) H \subset \mathscr{M}_{n}(H), \sigma(R) H$ and therefore, also $\sigma(R)$ will have a homomorphic image $\mathfrak{A}$ which is central simple algebra of $\operatorname{dim}<n^{2}$ and by passing to a splitting field of $\mathfrak{2}$ we will obtain an irreducible representation of $R$ of lower dimension which contradicts our assumption. Hence, $\sigma(R)=0$. This in turn implies that all $\sigma(r)_{i k} \in \mathfrak{q}$, but then $S_{0} \subseteq \mathfrak{q}$ since $S_{0}$ is generated by $\left\{\sigma(r)_{i k} ; r \in R\right\}$. Thus we have shown that $S_{0} / T_{0}$ contains no proper prime ideals from which we conclude that $S_{0} / T_{0}$ is a nil ring and in fact locally nilpotent as the lower radical which is the intersection of all prime ideals 
is in our case the whole ring. Consequently, also the matrix ring $\mathscr{M}_{n}\left(S_{0} / T_{0}\right)$ is locally nilpotent. On the other hand, we also have $\rho_{0}(R) S \supseteqq$ the image of $k^{0}[X] \Delta \bmod P$ and, therefore $\rho_{0}(R) S \supseteqq \mathscr{M}_{n}\left(T_{0}\right)$ since $k^{0}[X] \Delta \supseteqq \mathscr{M}_{n}(T)$.

Consider now first the case that $1 \in R$. Then $\rho_{0}(1)$ is an idempotent and therefore its image in $\mathscr{M}_{n}\left(S_{0} / T_{0}\right)$, which is nil, must be zero, i.e. $\rho_{0}(1) \in$ $\mathscr{M}_{n}\left(T_{0}\right)$. This implies that $\rho_{0}(R)=\rho_{0}(R) \rho_{0}(1) \cong \mathscr{M}_{n}\left(T_{0}\right)$ since $\mathscr{M}_{n}\left(T_{0}\right)$ is an ideal.

In the second case, $R$ is finitely generated and, therefore, $\rho_{0}(R) / \mathscr{M}_{n}\left(T_{0}\right)$ is also finitely generated in $\mathscr{M}_{n}\left(S_{0} / T_{0}\right)$ and therefore, it is nilpotent. This is possible in view of the fact that $R^{2}=R$ only if $\rho_{0}(R) \cong \mathscr{M}_{n}\left(T_{0}\right)$.

Thus, in both cases $\rho_{0}(R) \cong \mathscr{M}_{n}\left(T_{0}\right)$ and as the entries of $\rho_{0}(R)$ generates $S_{0}$ we must have $S_{0} \subseteq T_{0}$, and so $S_{0}=T_{0}$. Finally, we have shown that $\rho_{0}(R) S$ . $\mathscr{M}_{n}\left(T_{0}\right)$ which proves now that $\rho_{0}(R) S=\mathscr{M}_{n}\left(S_{0}\right)$.

Note that as $S=k+S_{0}$, the last result stated with $S_{0}$, only says that the algebra generated by $\rho_{0}(R)$ and $S_{0}$ is $\mathscr{M}_{n}\left(S_{0}\right)$.

Conversely, assume that $\rho_{0}(R) S=\mathscr{M}_{n}\left(S_{0}\right)$ and let $\alpha: R \rightarrow \mathscr{M}_{m}(K)$, for some field $K$, be a representation of $R$ of $\operatorname{dim} m<n$. Embed $\mathscr{M}_{m}(K) \cong \mathscr{M}_{n}(K)$ by putting the $m \times m$ matrices of $\mathscr{M}_{m}(K)$ in the upper corner of $\mathscr{M}_{n}(K)$ and we get an $n$ dimensional representation $\alpha_{0}: R \rightarrow \mathscr{M}_{n}(K)$. Theorem 1 implies that $\left(\mathscr{M}_{n} \eta\right) \rho_{0}=\alpha_{0}$ for some $\eta: S_{0} \rightarrow K$, but then $\alpha_{0}(R)=\left(\mathscr{M}_{n} \eta\right) \rho_{0}(R)$. Since $\rho_{0}(R) S$ $=\mathscr{M}_{n}\left(S_{0}\right)$ it follows that $\left(\mathscr{M}_{n} \eta\right)\left[\rho_{0}(R) S\right]$, which is $\alpha_{0}(R)$, will contain $n^{2}$ basis elements of $\mathscr{M}_{n}(K)$ unless $\eta\left(S_{0}\right)=0$. This must be the case, since $\alpha_{0}(R) \subseteq$ $\mathscr{M}_{m}(K)$ contains at most $m^{2}<n^{2}$ independent elements. This leads us to the conclusion that $\alpha(R)=0$, which means that $R$ has no representation of $\operatorname{dim}<n$.

If $R$ has a non zero representation $\alpha: R \rightarrow \mathscr{M}_{n}(K)$ with $\alpha \neq 0$, then preceding relations $\alpha(R)=\left(\mathscr{M}_{n} \eta\right) \rho_{0}(R)$ and $\rho_{0}(R) S=\mathscr{M}_{n}\left(S_{0}\right)$ imply that $S_{0} \neq 0$. Furthermore, the image $\alpha(R)$ over $K$ will generate over $K$ an algebra containing the ring $\mathscr{M}_{n}\left(\eta\left(S_{0}\right)\right)$ which has a base of $\mathscr{M}_{n}(K)$ since $\eta\left(S_{0}\right) \neq 0$ which proves that $\alpha$ is irreducible.

Finally, if $S_{0} \neq 0$, then the ring $S_{0}$ must contain a prime ideal, otherwise $S_{0}$ is a locally nilpotent ring which as before will imply in the cases of our theorem that $\rho_{0}(R)=0$. Since either $\rho_{0}(1) \in \rho_{0}(R)$ is an idempotent and so $\rho_{0}(1)=0$ which proves that $\rho_{0}(R)=\rho_{0}(R) \rho_{0}(1)=0$, or in the second case $R^{2}=R$, and $\rho_{0}(R)$ finitely generated in a locally nilpotent ring will imply that $\rho_{0}(R)$ $=0$. Now $\rho_{0}(R)=0$ implies by (a) of Theorem 1 that $S_{0}=0$, which is a contradiction. So we have shown that $S_{0}$ contains a prime ideal q. Let $\alpha$ be the composite map $R \rightarrow \mathscr{M}_{n}\left(S_{0}\right) \rightarrow \mathscr{M}_{n}\left(S_{0} / q\right) \rightarrow \mathscr{M}_{n}(K)$, where $K$ is the ring of quotients of $S_{0} / q$. Then that $\alpha$ is an irreducible representation follows from the facts that $\rho_{0}(R) S=\mathscr{M}_{n}\left(S_{0}\right)$ and $\mathscr{M}_{n}\left(S_{0} / \mathfrak{q}\right) K=\mathscr{M}_{n}(K)$.

COROLlary 4. Let $R \neq 0$ be a ring of the type given in Theorem 3 and which satisfies a polynomial identity of degree $\leqq 2 n$, then all irreducible repre- 
sentations of $R$ are of dimension $n$ if and only if $\rho_{0}(R) S=\mathscr{M}_{n}\left(S_{0}\right)$.

Proof. First we observe that in this case $R$ has a non-zero irreducible representation: for if $1 \in R$ or $R^{2}=R$ and finitely generated $R$ must contain a prime ideal $\mathfrak{q} \neq R$. Now $R / \mathfrak{q}$ is a prime ring satisfying a polynomial identity of degree $\leqq 2 n$, and hence its ring of quotient is a central simple algebra $D$ of $\operatorname{dim} \leqq n^{2}$ over its center. By extending the embedding of the ring of quotient to a complete matrix ring $\mathscr{M}_{n}(K)$, where $K$ is a splitting field of $D$ we get a non-zero representation $R \rightarrow R / \mathfrak{q} \rightarrow \mathscr{M}_{n}(K)$. The rest follows from the preceding theorem.

\section{$\S 5$. The unitary representations and Azumaya-algebras.}

Considering the category of ring with identity we obtained a universal embedding of [1], $\rho: R \rightarrow \mathscr{M}_{n}\left(S_{u}\right)$. For this embedding we have:

THEOREM 5. If all unitary representations of $R$ are of $\operatorname{dim} \geqq n$ then $\rho(R) S_{u}=\mathscr{M}_{n}\left(S_{u}\right)$ and in this case if $S_{u} \neq 0$ all unitary representations of $R$ of $\operatorname{dim} n$ are irreducible.

The proof of this theorem is the proof of Theorem 5 of [1] whose statement there is incorrect as the last part of that proof of embedding unitarily $\mathscr{M}_{m}(K) \cong \mathscr{M}_{n}(H)$ for $m<n$ can be carried out only for $m \mid n$. The corrected statement and its proof are the proof given in [1] to Theorem 5 with replacing $k^{0}[X]$ by $k[X]$.

In the special case $R$ is a central separable*) (an Azumaya) algebra of rank $n^{2}$, all representations of $R$ are of the form $R / \mathfrak{m} R$ where $\mathfrak{m}$ ranges over the maximal ideals of the center of $R$. Each $R / \mathfrak{m} R$ is a central simple of $\operatorname{dim} n^{2}$. By assumption there are no representation of lower dimension, hence our theorem implies that $\rho(R) S_{u}=\mathscr{M}_{n}\left(S_{u}\right)$. The algebra $R$ can be embedded in $n \times n$ matrix ring over a commutative ring and hence $\rho$ is a monomorphism. Finally, $\rho(R)$ is also an Azumaya algebra and, therefore, $\rho(R) \otimes S_{u} \cong \rho(R) S_{u}$ $=\mathscr{M}_{n}\left(S_{u}\right)$ where the tensor product is taken with respect to the center. Thus we have shown:

COROLlaRy 6. If $R$ is an Azumaya algebra of rank $n^{2}$ then $S_{u}$ is a splitting ring of $R$, and a commutative ring $K$ satisfies $R \otimes K \cong \mathscr{M}_{n}(K)$ if and only if $K$ contains a homomorphic image $K_{0}$ of $S_{u}$.

The first part of the corollary has just been shown. The second part follows from the universal property of $S_{u}$; since if $R \otimes K \cong \mathscr{M}_{n}(K)$ then we have the embedding $R \rightarrow R \otimes 1 \subseteq \mathscr{M}_{n}(K)$ which yields a homomorphism $\eta: S_{u}$ $\rightarrow K$, and thus $\eta\left(S_{u}\right)=K_{0} \subseteq K$. Conversely, since $\rho(R) S_{u}=\mathscr{M}_{n}\left(S_{u}\right)$ then any homomorphism $\eta: S_{u} \rightarrow K$ induces $\mathscr{M}_{n}(\eta): \mathscr{M}_{n}\left(S_{u}\right) \rightarrow \mathscr{M}_{n}(K)$. The composite

*) For results on Azumaya algebras see [2] and [3]. 
map $\sigma: R \rightarrow \mathscr{M}_{n}\left(S_{u}\right) \rightarrow \mathscr{M}_{n}(K)$ yields an Azumaya algebra $\sigma(R) \cong \mathscr{M}_{n}(K)$ with the center $\sigma(z)$ where $Z$ is the center of $R$. Hence, $\mathscr{M}_{n}(K)=\sigma(R) \underset{\sigma(z)}{\bigotimes_{(z)}} K \cong R \underset{z}{\bigotimes_{z} K}$ by considering $K$ as a $Z$-module by setting $z \cdot k=\sigma(z) k$ for every $k \in K, z \in Z$. q. e. d.

Hebrew University

Jerusalem, Israel

\section{Bibliography}

[1] S. A. Amitsur, Embeddings in Matrix rings, Pacific J. Math., 36 (1971), 21-29.

[2] H. Bass, Lectures on topics in algebraic $K$-theory, Tata Institute, Bombay, 1967.

[3] M. Auslander and O. Goldman, The Brauer group of a commutative ring, Trans. Amer. Math. Soc., 97 (1960). 